\title{
A Novel Insecticide Diafenthiuron 50WP against Cardamom Shoot and Capsule Borer $C$. punctiferalis Guenee
}

\author{
J. Aravind ${ }^{1 *}$, K. Samiayyan ${ }^{2}$ and S. Kuttalam ${ }^{2}$ \\ ${ }^{1}$ Department of Agricultural Entomology, TNAU, Coimbatore-641003, Tamil Nadu, India \\ ${ }^{2}$ Agricultural College and Research Intitute, Eachangottai, Thanjavur, Tamil Nadu, India \\ *Corresponding author
}

\section{A B S T R A C T}

Keywords

Diafenthiuron 50WP, Njellani gold, C. punctiferalis, IGR, phytotoxic symptoms

\section{Article Info}

Accepted: xx September 2017 Available Online: xx October 2017
Diafenthiuron, a novel insecticide with different mode of action of energy production inhibition and IGR activity was found to be effective against Conogethes punctiferalis in field trials conducted and concluded that 800ga.i./ha concentration can be recommended. Supervised field experiments that conducted during January 2014 - April 2014 at Nedukandam, Idukki district, Kerala with the cardamom cultivars, Njellani (Green gold) revealed that diafenthiuron $50 \mathrm{WP}$ (NS) as foliar application at 1600, 800 and $400 \mathrm{~g}$ a.i. ha ${ }^{-1}$ effected 77.07, 79.23 and 72.71 per cent reduction in capsule damage, and $77.86,77.63$ and 67.19 per cent reduction in shoot damage respectively, thirty days after three applications. No visual phytotoxic symptoms viz., leaf injury, wilting, vein clearing, necrosis, epinasty and hyponasty were observed with diafenthiuron 50 WP (NS) at 1600, 800 and 400 g. a.i. ha ${ }^{-1}$.

\section{Introduction}

Cardamom, the Queen of all spices, has a history as old as human race. It is the dried fruit of a herbaceous perennial plant. Warm humid climate, loamy soil rich in organic matter, distributed rainfall and special cultivation and processing methods all combine to make Indian cardamom truly unique-in aroma, flavour, size and it has parrot green colour. The main harvest season of cardamom in India is between JulyFebruary. About 72 insect pests have been recorded on cardamom (Chakravarthy and Khan, 1987). The major pests of cardamom are thrips Sciothrips cardamomi Ramk, capsule borers viz., lycaenid borer Jamides sp, scolytid borer Thamurgides cardamomi Schaufuss, shoot/panicle/capsule borer Conogethes punctiferalis Guenee, hairy caterpillars viz., Eupterote cardamomi Renga, Eupterote canaraica Moore, Eupterote fabia (Cramer), Eupterote testacea Walk., Lenodera vittata Walk. and Pericallia ricini (Fab.), Apart from these pests, shoot fly, Formosina flavipes Mall., rhizome weevil, Prodioctes haematicus Chevr., root borer, Hilarographa caminodes Meyer, whiteflies, Dialeurodes cardamomi (David and Subr.), aphids, Pentalonia nigronervosa Coq., scales, mealy bugs and mites have been found to affect the crop in various seasons (Kumaresan 
et al., 1987). Cardamom shoot and capsule borer (CSCB), C. punctiferalis has been an economic pest which feeds on the capsules, pseudostems and panicles causing more than 10 per cent yield loss under field conditions (Thyagaraj, 2002).

Diafenthiuron, a thio urea compound has a novel mode of action which inhibits and enhances biochemical sites such as respiration (Ishaaya et al., 2001); inhibits mitochondrial action and energy metabolism (ATP synthesis) and moult inhibition and hence it is seen as a viable tool for managing insects and mites. A seven year study (2000-2007) made to analyse the variability, possible trends in the multiplicity and management of cardamom pests, climatic variables and productivity in cardamom based agroforestry system showed a trend of decreasing maximum temperature since 2000 and a reduction in the incidence of insect pests along with a decrease in natural enemy population due to calendar-based pesticide spraying (Murugan et al., 2011).

\section{Materials and Methods}

A Field experiment was conducted at Nedukandam, Idukki district of kerela during January 2014 - April 2014 to evaluate the bioefficacy of diafenthiuron 50\%WP (NS), applied as foliar spray against cardamom shoot and capsule borer, C. punctiferalis and its phytotoxicity. Field trials were laid out in randomized block design (RBD) in the farmer's holdings in Nedukandam, Kerela on the ruling variety of Njellani Green Gold as per the treatments given below and replicated thrice. Three sprays were given at 30 days interval and observations were made on the capsule damage, before the spray and on 10, 20 and 30 days after application. Though the damage caused by borer in shoots and capsules the incidence was assessed on capsule basis and expressed as per cent damage. Damage was assessed by counting total number of capsules per ten panicles in four clumps in a treatment and capsules showing bored holes for borer and scabs for thrips on 10, 20 and 30 days after each application to find the per cent damage. The per cent damage was subjected to statistical analysis adopting randomized block design using AGRES after converting it to arcsine percentage

\section{Phytotoxicity tests for diafenthiuron}

To evaluate the phytotoxicity (if any) caused by diafenthiuron $50 \% \mathrm{WP}$ on cardamom, field experiment was conducted at Nedukandam, Kerela during December 2012 to March 2013. The experiments were conducted in a randomized block design with 4 clumps per treatment and with three replications using the variety Njellani Green Gold. Three different doses of 800,1600 , and $3200 \mathrm{~g}$ a.i. ha ${ }^{-1}$. The plants were observed on 1, 3, 7, 10, 14 and 20 days after spraying as per the protocol of Central Insecticide Board Registration Committee (C.I.B.R.C) for the phytotoxic symptoms like Injury to leaftip and leafsurface,Wilting, Vein clearing, Necrosis, Epinasty and hyponasty.

The per cent leaf injury was calculated using the formula,

Total grade points
Per cent leaf injury =
Max. Grade x No. of leaves observed

\section{Results and Discussion}

The results of field and laboratory experiments conducted to assess the bioefficacy of diafenthiuron 50WP (NS) against the cardamom shoot and capsule borer and its its phytotoxicity. The mean damage to capsules by CSCB before application ranged between 12.20 and 12.80 per cent (Table 1) and was not significant. After first spray, at ten days after application (DAA), reduction in damage was noticed in treated plots (12.20 to 
$12.80 \%)$ while the untreated control registered 12.20 per cent. At 20 DAA, diafenthiuron 50 WP (NS) at the highest dose of $1600 \mathrm{~g}$ a.i. ha ${ }^{-1}$ recorded the lowest of 12.00 per cent damage to capsules which was on compared with its lower dose of $400 \mathrm{~g}$ a.i. $\mathrm{ha}^{-1}(12.40 \%)$ and diafenthiuron $50 \mathrm{WP}$ (ES) $800 \mathrm{~g}^{2}$.i. $\mathrm{ha}^{-1}(12.05 \%)$. The standard checks, quinalphos $25 \mathrm{EC} 600 \mathrm{~g}$ a.i. $\mathrm{ha}^{-1}$ and $1200 \mathrm{~g}$ a.i. ha ${ }^{-1}$ recorded $18.00,11.75$ and 11.75 per cent damage. At 30 DAA, diafenthiuron 50 WP (NS) $1600 \mathrm{~g}$ a.i. ha ${ }^{-1}$ recorded 11.55 per cent damage to capsules and was on par with lower dose of $800 \mathrm{~g}$ a.i. ha ${ }^{-1}$ which recorded 11.70 per cent damage and diafenthiuron 50 WP (ES) $800 \mathrm{~g}$ a.i. ha ${ }^{-1}(11.90 \%)$. The standard checks, quinalphos 25 EC $600 \mathrm{~g}$ a.i. $\mathrm{ha}^{-1}$ and quinalphos 25 EC $1200 \mathrm{~g}$ a.i. $\mathrm{ha}^{-1}$ recorded 11.70 and 11.55 per cent damage respectively while untreated check recorded the highest of 21.50 per cent. The per cent reduction over check obtained maximum in diafenthiuron 50 WP 1600 g a.i. ha ${ }^{-1}$ $(30.26 \%)$ followed by standard quinalphos $25 \%$ EC at $1200 \mathrm{~g}$ a i. ha ${ }^{-1}$ (Table $1 \&$ Fig 1$)$
The second application was given thirty days after the first application. The trend in efficacy of different treatments in respect of per cent reduction in borer damage was similar to that of first application (Table $2 \&$ Fig 2). Diafenthiuron 50 WP (NS) 1600 and $800 \mathrm{~g}$ a.i. $\mathrm{ha}^{-1}$ recorded $66.58 \%$ and $64.03 \%$ after 30 Days after first application.

The third spray was taken up thirty days after the second application, when the damage level reached 8.25 to 9.00 per cent in the treatments and 29.00 per cent in untreated check. Trend in reduction of capsule damage continued to be similar as that of second application. Based on reduction of capsule damage over untreated check, the descending order of efficacy of different treatments are: diafenthiuron 50 WP (NS) $800 \mathrm{~g}$ a.i. ha ${ }^{-1}$ $(79.23 \%), \quad 1600 \mathrm{~g}$ a.i. ha $^{-1}(77.07 \%)$, quinalphos $25 \mathrm{EC} 1200 \mathrm{~g}$ a.i. $\mathrm{ha}^{-1}(76.96 \%)$, quinalphos 25 EC $600 \mathrm{~g}$ a.i. $\mathrm{ha}^{-1}(76.19)$, diafenthiruon 50 WP ES $800 \mathrm{~g}$ a.i. $\mathrm{ha}^{-1}(76.41$ $\%$ ), diafenthiruon 50 WP (NS) 400 g a.i. ha ${ }^{-1}$ (72.71) (Table 3 \& Fig 3).

Table.1 Effect of diafenthiuron 50\% WP (NS) on capsule damage by C. punctiferalis (Location - Nedukandam) - First Application

\begin{tabular}{|c|c|c|c|c|c|c|c|}
\hline \multirow[b]{2}{*}{ Treatments } & \multirow{2}{*}{$\begin{array}{c}\text { Dose } \\
\left(\mathrm{g} \text { a.i. } \mathbf{h a}^{-1}\right)\end{array}$} & \multicolumn{6}{|c|}{ Per cent capsule damage } \\
\hline & & PTC & 10DAA & 20DAA & 30DAA & Mean & $\begin{array}{c}\text { Percent reduction } \\
\text { over check }\end{array}$ \\
\hline $\begin{array}{l}\text { Diafenthiuron } 50 \% \text { WP NS } \\
\left(1.6 \mathrm{~g} \mathrm{~L}^{-1}\right)\end{array}$ & 400 & 12.50 & $\begin{array}{l}12.50^{\mathrm{ab}} \\
(20.70)\end{array}$ & $\begin{array}{l}12.40^{\mathrm{ab}} \\
(20.61)\end{array}$ & $\begin{array}{l}12.15^{b} \\
(20.39)\end{array}$ & 12.35 & 28.32 \\
\hline $\begin{array}{l}\text { Diafenthiuron 50\% WP NS (3.2 } \\
\left.\mathrm{g} \mathrm{L}^{-1}\right)\end{array}$ & 800 & 12.63 & $\begin{array}{l}12.63^{\mathrm{b}} \\
(20.81)\end{array}$ & $\begin{array}{l}12.00^{\mathrm{b}} \\
(20.26)\end{array}$ & $\begin{array}{l}11.70^{\mathrm{ab}} \\
(20.01)\end{array}$ & 12.11 & 29.72 \\
\hline $\begin{array}{l}\text { Diafenthiuron 50\% WP NS (6.4 } \\
\left.\mathrm{g} \mathrm{L}^{-1}\right)\end{array}$ & 1600 & 12.45 & $\begin{array}{l}12.45^{\mathrm{c}} \\
(20.66)\end{array}$ & $\begin{array}{l}12.05^{\mathrm{d}} \\
(20.31)\end{array}$ & $\begin{array}{l}11.55^{\mathrm{a}} \\
(19.86)\end{array}$ & 12.02 & 30.26 \\
\hline $\begin{array}{l}\text { Standard Diafenthiuron } 50 \% \\
\text { WP }\left(3.2 \mathrm{~g} \mathrm{~L}^{-1}\right)\end{array}$ & 800 & 12.40 & $\begin{array}{l}12.40^{b} \\
(20.61)\end{array}$ & $\begin{array}{l}11.95^{b} \\
(20.22)\end{array}$ & $\begin{array}{l}11.90^{\mathrm{ab}} \\
(20.17)\end{array}$ & 12.08 & 29.87 \\
\hline $\begin{array}{l}\text { Standard Quinalphos 25\% EC } \\
\left(7.2 \mathrm{ml} \mathrm{L}^{-1}\right)\end{array}$ & 600 & 12.68 & $\begin{array}{l}12.68^{\mathrm{ab}} \\
(20.86)\end{array}$ & $\begin{array}{l}11.75^{\mathrm{ab}} \\
(20.04)\end{array}$ & $\begin{array}{l}11.70^{\mathrm{ab}} \\
(20.01)\end{array}$ & 12.04 & 30.10 \\
\hline $\begin{array}{l}\text { Standard Quinalphos 25\% EC } \\
\left(14.4 \mathrm{ml} \mathrm{L}^{-1}\right)\end{array}$ & 1200 & 12.80 & $\begin{array}{l}12.80^{\mathrm{ab}} \\
(20.96)\end{array}$ & $\begin{array}{l}11.75^{a b} \\
(20.04)\end{array}$ & $\begin{array}{l}11.55^{\mathrm{ab}} \\
(19.86)\end{array}$ & 12.03 & 30.16 \\
\hline Untreated check & - & 12.20 & $\begin{array}{l}12.20^{\mathrm{c}} \\
(20.44)\end{array}$ & $\begin{array}{l}18.00^{\mathrm{c}} \\
(25.14)\end{array}$ & $\begin{array}{l}21.50^{\mathrm{c}} \\
(27.62)\end{array}$ & 17.23 & \\
\hline
\end{tabular}

Mean of three observations; PTC - Pretreatment count,

Figures in parentheses are arc sin transformed values, in a column, means followed by a common letter(s) are not significantly different by LSD $(\mathrm{P}=0.05)$ 
Table.2 Effect of diafenthiuron 50\% WP (NS) on capsule damage by $C$. punctiferalis (Location - Nedukandam) - Second Application

\begin{tabular}{|c|c|c|c|c|c|c|c|}
\hline \multirow[b]{2}{*}{ Treatments } & \multirow{2}{*}{$\begin{array}{c}\text { Dose } \\
\left(\mathrm{g} \text { a.i. } \text { ha }^{-1}\right)\end{array}$} & \multicolumn{6}{|c|}{ Per cent capsule damage } \\
\hline & & PTC & 10 DAA & 20 DAA & 30 DAA & Mean & $\begin{array}{c}\text { Percent } \\
\text { reduction } \\
\text { over } \\
\text { check }\end{array}$ \\
\hline $\begin{array}{l}\text { Diafenthiuron } 50 \% \text { WP NS } \\
\left(1.6 \mathrm{~g} \mathrm{~L}^{-1}\right)\end{array}$ & 400 & 12.15 & $\begin{array}{l}11.15^{\mathrm{b}} \\
(19.50)\end{array}$ & $\begin{array}{l}10.50^{\mathrm{b}} \\
(18.90)\end{array}$ & $\begin{array}{l}10.00^{\mathrm{c}} \\
(18.43)\end{array}$ & 10.55 & 60.68 \\
\hline $\begin{array}{l}\text { Diafenthiuron 50\% WP NS } \\
\left(3.2 \mathrm{~g} \mathrm{~L}^{-1}\right)\end{array}$ & 800 & 11.70 & $\begin{array}{l}10.20^{\mathrm{ab}} \\
(18.62)\end{array}$ & $\begin{array}{l}9.75^{\mathrm{ab}} \\
(18.19)\end{array}$ & $\begin{array}{l}9.00^{\mathrm{bc}} \\
(17.45)\end{array}$ & 9.65 & 64.03 \\
\hline $\begin{array}{l}\text { Diafenthiuron 50\% WP NS } \\
\left(6.4 \mathrm{~g} \mathrm{~L}^{-1}\right)\end{array}$ & 1600 & 11.55 & $\begin{array}{l}9.65^{\mathrm{a}} \\
(18.09)\end{array}$ & $\begin{array}{l}9.00^{\mathrm{a}} \\
(17.45)\end{array}$ & $\begin{array}{l}8.25^{\mathrm{a}} \\
(16.69)\end{array}$ & 8.97 & 66.58 \\
\hline $\begin{array}{l}\text { Standard Diafenthiuron } 50 \% \mathrm{WP} \\
\left(3.2 \mathrm{~g} \mathrm{~L}^{-1}\right)\end{array}$ & 800 & 11.90 & $\begin{array}{c}9.45^{\mathrm{ab}} \\
(17.90)\end{array}$ & $\begin{array}{c}8.85^{\mathrm{a}} \\
(17.30)\end{array}$ & $\begin{array}{l}8.65^{\text {bc }} \\
(17.10)\end{array}$ & 8.98 & 66.52 \\
\hline $\begin{array}{l}\text { Standard Quinalphos } 25 \% \text { EC } \\
\left(7.2 \mathrm{ml} \mathrm{L}^{-1}\right)\end{array}$ & 600 & 11.70 & $\begin{array}{l}9.75^{\mathrm{ab}} \\
(18.19)\end{array}$ & $\begin{array}{l}9.10^{\mathrm{ab}} \\
(17.55)\end{array}$ & $\begin{array}{l}8.85^{\text {bc }} \\
(17.30)\end{array}$ & 9.23 & 65.59 \\
\hline $\begin{array}{l}\text { Standard Quinalphos 25\% EC } \\
\left(14.4 \mathrm{ml} \mathrm{L}^{-1}\right)\end{array}$ & 1200 & 11.55 & $\begin{array}{l}9.80^{\mathrm{ab}} \\
(18.24)\end{array}$ & $\begin{array}{l}8.95^{\mathrm{ab}} \\
(17.40)\end{array}$ & $\begin{array}{l}8.90^{\mathrm{b}} \\
(17.35)\end{array}$ & 9.22 & 65.65 \\
\hline Untreated check & - & 21.50 & $\begin{array}{l}24.00^{c} \\
(29.33)\end{array}$ & $\begin{array}{l}27.50^{c} \\
(31.62)\end{array}$ & $\begin{array}{l}29.00^{\mathrm{d}} \\
(31.62)\end{array}$ & 26.83 & \\
\hline
\end{tabular}

Mean of three observations; PTC - Pretreatment count,

Figures in parentheses are arc sin transformed values.

In a column, means followed by a common letter(s) are not significantly different by LSD ( $\mathrm{P}=0.05)$

Table.3 Effect of diafenthiuron 50\% WP (NS) on capsule damage by $C$. punctiferalis (Location - Nedukandam) - Third Application

\begin{tabular}{|c|c|c|c|c|c|c|c|}
\hline \multirow[b]{2}{*}{ Treatments } & \multirow{2}{*}{$\begin{array}{c}\text { Dose } \\
\left(\mathrm{g} \text { a.i. } \text { ha }^{-1}\right)\end{array}$} & \multicolumn{6}{|c|}{ Per cent capsule damage } \\
\hline & & PTC & 10 DAA & 20 DAA & 30 DAA & Mean & $\begin{array}{c}\text { Percent } \\
\text { reduction } \\
\text { over check }\end{array}$ \\
\hline $\begin{array}{l}\text { Diafenthiuron } 50 \% \text { WP NS } \\
\left(1.6 \mathrm{~g} \mathrm{~L}^{-1}\right)\end{array}$ & 400 & 10.00 & $\begin{array}{l}8.50^{\mathrm{c}} \\
(16.95)\end{array}$ & $\begin{array}{l}8.25^{\mathrm{c}} \\
(16.69)\end{array}$ & $\begin{array}{c}7.95^{\mathrm{c}} \\
(16.37)\end{array}$ & 8.23 & 72.71 \\
\hline $\begin{array}{l}\text { Diafenthiuron 50\% WP NS } \\
\left(3.2 \mathrm{~g} \mathrm{~L}^{-1}\right)\end{array}$ & 800 & 9.00 & $\begin{array}{c}6.90^{\mathrm{b}} \\
(15.22)\end{array}$ & $\begin{array}{c}6.00^{\mathrm{b}} \\
(14.17)\end{array}$ & $\begin{array}{c}5.90^{\mathrm{b}} \\
(14.05)\end{array}$ & 6.27 & 79.23 \\
\hline $\begin{array}{l}\text { Diafenthiuron 50\% WP NS } \\
\left(6.4 \mathrm{~g} \mathrm{~L}^{-1}\right)\end{array}$ & 1600 & 8.25 & $\begin{array}{c}7.25^{\mathrm{a}} \\
(15.62)\end{array}$ & $\begin{array}{c}6.80^{\mathrm{a}} \\
(15.11)\end{array}$ & $\begin{array}{c}6.70^{\mathrm{a}} \\
(15.00)\end{array}$ & 6.92 & 77.07 \\
\hline $\begin{array}{l}\text { Standard Diafenthiuron 50\% WP } \\
\left(3.2 \mathrm{~g} \mathrm{~L}^{-1}\right)\end{array}$ & 800 & 8.65 & $\begin{array}{c}7.25^{\mathrm{bc}} \\
(15.62)\end{array}$ & $\begin{array}{c}7.10^{\mathrm{b}} \\
(15.43)\end{array}$ & $\begin{array}{c}7.00^{\mathrm{b}} \\
(15.34)\end{array}$ & 7.12 & 76.41 \\
\hline $\begin{array}{l}\text { Standard Quinalphos 25\% EC } \\
\left(7.2 \mathrm{ml} \mathrm{L}^{-1}\right)\end{array}$ & 600 & 8.85 & $\begin{array}{c}7.60^{\mathrm{b}} \\
(16.00)\end{array}$ & $\begin{array}{c}7.10^{\mathrm{b}} \\
(15.45)\end{array}$ & $\begin{array}{c}6.85^{\mathrm{b}} \\
(15.17)\end{array}$ & 7.18 & 76.19 \\
\hline $\begin{array}{l}\text { Standard Quinalphos 25\% EC } \\
\left(14.4 \mathrm{ml} \mathrm{L}^{-1}\right)\end{array}$ & 1200 & 8.90 & $\begin{array}{c}7.20^{\mathrm{b}} \\
(15.56)\end{array}$ & $\begin{array}{c}6.90^{\mathrm{b}} \\
(15.22)\end{array}$ & $\begin{array}{l}6.75^{\mathrm{bc}} \\
(15.05)\end{array}$ & 6.95 & 76.96 \\
\hline Untreated check & - & 29.00 & $\begin{array}{l}32.00^{\mathrm{d}} \\
(34.44)\end{array}$ & $\begin{array}{l}30.00^{\mathrm{d}} \\
(33.21)\end{array}$ & $\begin{array}{l}28.50^{\mathrm{d}} \\
(32.26)\end{array}$ & 30.17 & \\
\hline
\end{tabular}

Mean of three observations PTC- Pretreatment count DAA -Days after application

Figures in parentheses are arc sin transformed values

In a column, means followed by a common letter(s) are not significantly different by LSD ( $\mathrm{P}=0.05)$ 
Table.4 Effect of diafenthiuron 50\% WP (NS) on shoot damage by $C$. punctiferalis (Location - Nedukandam) - First Application

\begin{tabular}{|c|c|c|c|c|c|c|c|}
\hline \multirow[b]{2}{*}{ Treatments } & \multirow{2}{*}{$\begin{array}{c}\text { Dose } \\
\left(\mathrm{g} \text { a.i. } \mathbf{h a}^{-1}\right)\end{array}$} & \multicolumn{6}{|c|}{ Per cent shoot damage } \\
\hline & & PTC & $\begin{array}{c}\text { 10 } \\
\text { DAA }\end{array}$ & $\begin{array}{c}20 \\
\text { DAA }\end{array}$ & $\begin{array}{c}\text { 30 } \\
\text { DAA }\end{array}$ & Mean & $\begin{array}{l}\text { Percent reduction } \\
\text { over check }\end{array}$ \\
\hline $\begin{array}{l}\text { Diafenthiuron } 50 \% \text { WP NS } \\
\left(1.6 \mathrm{~g} \mathrm{~L}^{-1}\right)\end{array}$ & 400 & 18.52 & $\begin{array}{l}18.52^{\mathrm{abc}} \\
(25.48)\end{array}$ & $\begin{array}{l}18.00^{\mathrm{ab}} \\
(25.10)\end{array}$ & $\begin{array}{l}18.00^{\mathrm{c}} \\
(25.10)\end{array}$ & 18.17 & 22.50 \\
\hline $\begin{array}{l}\text { Diafenthiuron 50\% WP NS } \\
\left(3.2 \mathrm{~g} \mathrm{~L}^{-1}\right)\end{array}$ & 800 & 16.90 & $\begin{array}{l}16.90^{\mathrm{a}} \\
(24.27)\end{array}$ & $\begin{array}{l}16.25^{\mathrm{a}} \\
(23.77)\end{array}$ & $\begin{array}{l}16.00^{\mathrm{ab}} \\
(23.57)\end{array}$ & 16.38 & 30.14 \\
\hline $\begin{array}{l}\text { Diafenthiuron 50\% WP NS } \\
\left(6.4 \mathrm{~g} \mathrm{~L}^{-1}\right)\end{array}$ & 1600 & 16.95 & $\begin{array}{l}16.95^{\mathrm{ab}} \\
(24.31)\end{array}$ & $\begin{array}{l}16.70^{\mathrm{a}} \\
(24.12)\end{array}$ & $\begin{array}{l}14.90^{\mathrm{a}} \\
(22.70)\end{array}$ & 16.18 & 30.99 \\
\hline $\begin{array}{l}\text { Standard Diafenthiuron 50\% } \\
\text { WP }\left(3.2 \mathrm{~g} \mathrm{~L}^{-1}\right)\end{array}$ & 800 & 18.50 & $\begin{array}{l}18.50^{\mathrm{abc}} \\
(25.47)\end{array}$ & $\begin{array}{l}18.00^{\mathrm{ab}} \\
(25.10)\end{array}$ & $\begin{array}{l}17.00^{\mathrm{bc}} \\
(24.35)\end{array}$ & 17.00 & 27.51 \\
\hline $\begin{array}{l}\text { Standard Quinalphos 25\% EC } \\
\left(7.2 \mathrm{ml} \mathrm{L}^{-1}\right)\end{array}$ & 600 & 20.00 & $\begin{array}{l}20.00^{c} \\
(26.56)\end{array}$ & $\begin{array}{l}19.00^{\mathrm{b}} \\
(25.84)\end{array}$ & $\begin{array}{l}17.50^{\mathrm{c}} \\
(24.72)\end{array}$ & 18.83 & 19.69 \\
\hline $\begin{array}{l}\text { Standard Quinalphos 25\% EC } \\
\left(14.4 \mathrm{ml} \mathrm{L}^{-1}\right)\end{array}$ & 1200 & 16.50 & $\begin{array}{l}16.50^{\mathrm{a}} \\
(23.96)\end{array}$ & $\begin{array}{l}16.20^{\mathrm{a}} \\
(23.73)\end{array}$ & $\begin{array}{l}16.10^{\mathrm{ab}} \\
(23.65)\end{array}$ & 16.27 & 30.63 \\
\hline Untreated check & - & 22.25 & $\begin{array}{l}18.90^{\mathrm{bc}} \\
(28.14)\end{array}$ & $\begin{array}{l}22.50^{\mathrm{c}} \\
(28.31)\end{array}$ & $\begin{array}{l}25.60^{\mathrm{d}} \\
(30.39)\end{array}$ & 23.45 & \\
\hline
\end{tabular}

Mean of three observations PTC- Pretreatment count DAA -Days after application

Figures in parentheses are arc sin transformed values

In a column, means followed by a common letter(s) are not significantly different by LSD ( $\mathrm{P}=0.05)$

Table.5 Effect of diafenthiuron 50\% WP (NS) on shoot damage by $C$. punctiferalis (Location - Nedukandam) - Second Application

\begin{tabular}{|c|c|c|c|c|c|c|c|}
\hline \multirow[b]{2}{*}{ Treatments } & \multirow{2}{*}{$\begin{array}{c}\text { Dose } \\
\left(\mathrm{g} \text { a.i. } \text { ha }^{-1}\right)\end{array}$} & \multicolumn{6}{|c|}{ Per cent shoot damage } \\
\hline & & PTC & $\begin{array}{c}\text { 10 } \\
\text { DAA }\end{array}$ & $\begin{array}{c}20 \\
\text { DAA }\end{array}$ & $\begin{array}{c}\text { 30 } \\
\text { DAA }\end{array}$ & Mean & $\begin{array}{c}\text { Percent reduction over } \\
\text { check }\end{array}$ \\
\hline $\begin{array}{l}\text { Diafenthiuron } 50 \% \text { WP NS } \\
\left(1.6 \mathrm{~g} \mathrm{~L}^{-1}\right)\end{array}$ & 400 & 18.00 & $\begin{array}{l}16.50^{\mathrm{d}} \\
(30.98)\end{array}$ & $\begin{array}{l}15.50^{\mathrm{d}} \\
(30.32)\end{array}$ & $\begin{array}{l}14.80^{\mathrm{d}} \\
(29.86)\end{array}$ & 15.60 & 49.49 \\
\hline $\begin{array}{l}\text { Diafenthiuron 50\% WP NS } \\
\left(3.2 \mathrm{~g} \mathrm{~L}^{-1}\right)\end{array}$ & 800 & 16.00 & $\begin{array}{l}14.50^{b} \\
(29.66)\end{array}$ & $\begin{array}{l}14.00^{\mathrm{bc}} \\
(29.33)\end{array}$ & $\begin{array}{l}12.25^{\mathrm{ab}} \\
(28.14)\end{array}$ & 13.58 & 56.16 \\
\hline $\begin{array}{l}\text { Diafenthiuron 50\% WP NS } \\
\left(6.4 \mathrm{~g} \mathrm{~L}^{-1}\right)\end{array}$ & 1600 & 14.90 & $\begin{array}{l}12.15^{\mathrm{a}} \\
(28.07)\end{array}$ & $\begin{array}{l}11.50^{\mathrm{a}} \\
(27.62)\end{array}$ & $\begin{array}{l}10.00^{\mathrm{a}} \\
(26.56)\end{array}$ & 11.22 & 64.00 \\
\hline $\begin{array}{l}\text { Standard Diafenthiuron } 50 \% \\
\text { WP }\left(3.2 \mathrm{~g} \mathrm{~L}^{-1}\right)\end{array}$ & 800 & 17.00 & $\begin{array}{l}14.50^{\mathrm{b}} \\
(29.66)\end{array}$ & $\begin{array}{l}14.25^{\mathrm{bc}} \\
(29.50)\end{array}$ & $\begin{array}{l}12.10^{\mathrm{bc}} \\
(28.72)\end{array}$ & 13.62 & 56.02 \\
\hline $\begin{array}{l}\text { Standard Quinalphos } 25 \% \text { EC } \\
\left(7.2 \mathrm{ml} \mathrm{L}^{-1}\right)\end{array}$ & 600 & 17.50 & $\begin{array}{l}15.20^{\mathrm{c}} \\
(30.13)\end{array}$ & $\begin{array}{l}14.20^{\mathrm{bc}} \\
(29.46)\end{array}$ & $\begin{array}{l}13.50^{\mathrm{bc}} \\
(28.99)\end{array}$ & 14.30 & 53.78 \\
\hline $\begin{array}{l}\text { Standard Quinalphos 25\% EC } \\
\left(14.4 \mathrm{ml} \mathrm{L}^{-1}\right)\end{array}$ & 1200 & 16.10 & $\begin{array}{l}14.00^{\mathrm{b}} \\
(29.33)\end{array}$ & $\begin{array}{l}13.50^{\mathrm{b}} \\
(28.99)\end{array}$ & $\begin{array}{l}13.00^{\mathrm{b}} \\
(28.82)\end{array}$ & 13.50 & 55.98 \\
\hline Untreated check & - & 25.60 & $\begin{array}{l}28.60^{\mathrm{e}} \\
(38.23)\end{array}$ & $\begin{array}{l}28.50^{\mathrm{e}} \\
(38.35)\end{array}$ & $\begin{array}{c}32.50^{\mathrm{e}} \\
(38.43)\end{array}$ & 29.87 & \\
\hline
\end{tabular}

Mean of three observations PTC- Pretreatment count DAA -Days after application

Figures in parentheses are arc sin transformed values

In a column, means followed by a common letter(s) are not significantly different by LSD $(\mathrm{P}=0.05)$ 
Table.6 Effect of diafenthiuron 50\% WP (NS) on shoot damage by $C$. punctiferalis (Location - Nedukandam) - Third Application

\begin{tabular}{|c|c|c|c|c|c|c|c|}
\hline \multirow[b]{2}{*}{ Treatments } & \multirow{2}{*}{$\begin{array}{c}\text { Dose } \\
\left(\mathrm{g} \text { a.i. } \text { ha }^{-1}\right)\end{array}$} & \multicolumn{6}{|c|}{ Per cent shoot damage } \\
\hline & & PTC & $\begin{array}{c}\text { 10 } \\
\text { DAA }\end{array}$ & $\begin{array}{c}20 \\
\text { DAA }\end{array}$ & $\begin{array}{c}\text { 30 } \\
\text { DAA } \\
\end{array}$ & Mean & $\begin{array}{c}\text { Percent reduction over } \\
\text { check }\end{array}$ \\
\hline $\begin{array}{l}\text { Diafenthiuron } 50 \% \text { WP NS } \\
\left(1.6 \mathrm{~g} \mathrm{~L}^{-1}\right)\end{array}$ & 400 & 14.80 & $\begin{array}{l}14.80 \\
(21.55) \\
\end{array}$ & $\begin{array}{l}13.50 \\
(20.26)\end{array}$ & $\begin{array}{c}12.00 \\
(19.18)\end{array}$ & 12.10 & 67.19 \\
\hline $\begin{array}{l}\text { Diafenthiuron 50\% WP NS } \\
\left(3.2 \mathrm{~g} \mathrm{~L}^{-1}\right)\end{array}$ & 800 & 12.25 & $\begin{array}{c}9.75^{\mathrm{b}} \\
(17.59)\end{array}$ & $\begin{array}{c}8.00^{\mathrm{b}} \\
(15.58)\end{array}$ & $\begin{array}{l}7.00^{\mathrm{b}} \\
(15.42)\end{array}$ & 8.25 & 77.63 \\
\hline $\begin{array}{l}\text { Diafenthiuron 50\% WP NS } \\
\left(6.4 \mathrm{~g} \mathrm{~L}^{-1}\right)\end{array}$ & 1600 & 10.00 & $\begin{array}{l}10.00^{\mathrm{a}} \\
(17.45)\end{array}$ & $\begin{array}{c}9.00^{\mathrm{a}} \\
(16.42)\end{array}$ & $\begin{array}{l}8.00^{\mathrm{a}} \\
(15.89)\end{array}$ & 8.17 & 77.86 \\
\hline $\begin{array}{l}\text { Standard Diafenthiuron 50\% WP } \\
\left(3.2 \mathrm{~g} \mathrm{~L}^{-1}\right)\end{array}$ & 800 & 12.10 & $\begin{array}{c}12.10 \\
(19.46) \\
\end{array}$ & $\begin{array}{l}11.10 \\
(17.95) \\
\end{array}$ & $\begin{array}{c}9.50 \\
(17.20) \\
\end{array}$ & 9.78 & 73.47 \\
\hline $\begin{array}{l}\text { Standard Quinalphos 25\% EC } \\
\left(7.2 \mathrm{ml} \mathrm{L}^{-1}\right)\end{array}$ & 600 & 13.50 & $\begin{array}{c}13.50 \\
(20.70) \\
\end{array}$ & $\begin{array}{c}12.50 \\
(19.36) \\
\end{array}$ & $\begin{array}{c}11.00 \\
(17.70) \\
\end{array}$ & 10.92 & 70.40 \\
\hline $\begin{array}{l}\text { Standard Quinalphos 25\% EC } \\
\left(14.4 \mathrm{ml} \mathrm{L}^{-1}\right)\end{array}$ & 1200 & 13.00 & $\begin{array}{c}13.00 \\
(20.26) \\
\end{array}$ & $\begin{array}{c}12.00 \\
(18.43) \\
\end{array}$ & $\begin{array}{c}10.00 \\
(17.45) \\
\end{array}$ & 10.33 & 71.98 \\
\hline Untreated check & - & 32.50 & $\begin{array}{l}32.50 \\
(35.36)\end{array}$ & $\begin{array}{l}33.50 \\
(38.35)\end{array}$ & $\begin{array}{c}38.50 \\
(38.43)\end{array}$ & 36.88 & \\
\hline
\end{tabular}

Mean of three observations; PTC - Pretreatment count DAA -Days after application

Figures in parentheses are arc sin transformed values

In a column, means followed by a common letter(s) are not significantly different by LSD ( $\mathrm{P}=0.05)$

Table.7 Phytotoxic effect of diafenthiuron $50 \% \mathrm{WP}$ on cardamom - Experiment I (Location- Nedukandam)

\begin{tabular}{|c|l|c|c|c|c|c|c|c|}
\hline \multirow{2}{*}{ S.No. } & \multirow{2}{*}{ Treatments } & \multirow{2}{*}{ Dose (\%) } & \multicolumn{6}{|c|}{ Phytotoxicity rating * } \\
\cline { 4 - 9 } & & & $\begin{array}{c}\text { Leaf } \\
\text { injury }\end{array}$ & Wilting & $\begin{array}{c}\text { Vein } \\
\text { clearing }\end{array}$ & Necrosis & Epinasty & Hyponasty \\
\hline 1. & Diafenthiuron50\%WP & 0.08 & 0 & 0 & 0 & 0 & 0 & 0 \\
\hline 2. & Diafenthiuron50\%WP & 0.16 & 0 & 0 & 0 & 0 & 0 & 0 \\
\hline 3. & Diafenthiuron50\%WP & 0.32 & 0 & 0 & 0 & 0 & 0 & 0 \\
\hline 4. & Untreated check & - & 0 & 0 & 0 & 0 & 0 & 0 \\
\hline
\end{tabular}

(Mean of five observations); Observed on 1, 3, 7, 10, 14 and 20 days after spraying

Fig.1 Effect of diafenthiuron 50\% WP (NS) on capsule damage by $C$. punctiferalis (Location - Nedukandam) - First Application

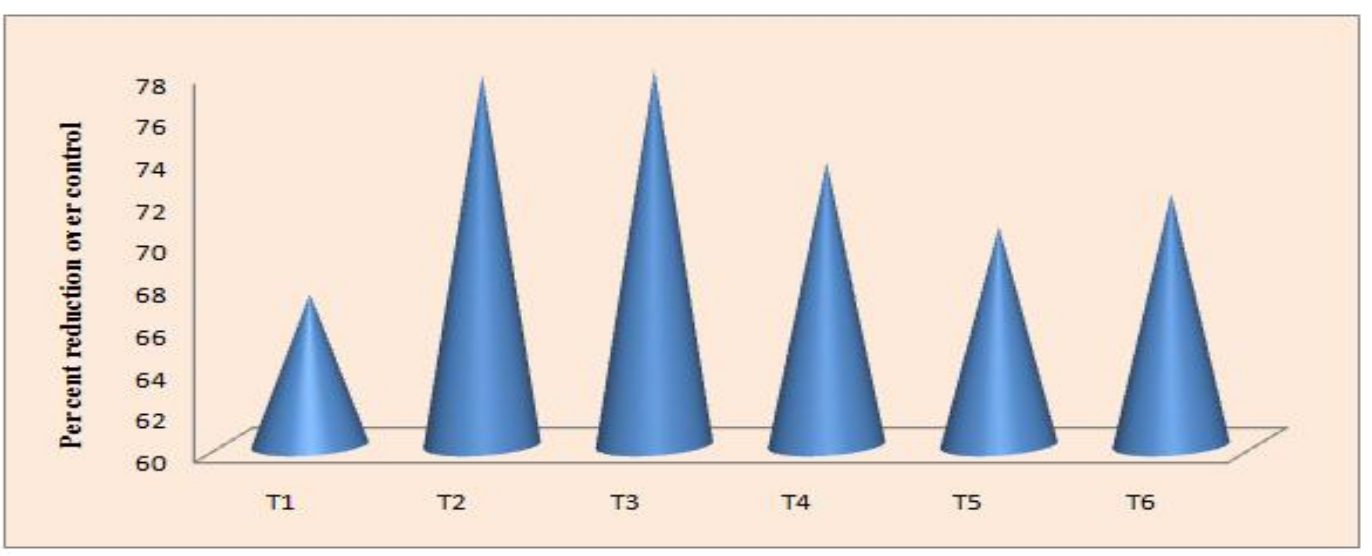

$\mathrm{T}_{1}$ - Diafenthiuron 50\%WP NS (1.6g L $\left.\mathrm{L}^{-1}\right), \mathrm{T}_{2}$ - Diafenthiuron 50\% WP NS $\left(3.2 \mathrm{~g} \mathrm{~L}^{-1}\right), \mathrm{T}_{3}$ - Diafenthiuron 50\% WP

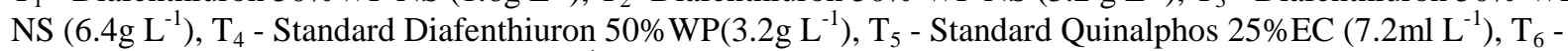
Standard Quinalphos $25 \%$ EC $\left(14.4 \mathrm{ml} \mathrm{L}^{-1}\right)$ 
Fig.2 Effect of diafenthiuron 50\% WP (NS) on capsule damage by $C$. punctiferalis (Location -Nedukandam) - Second Application

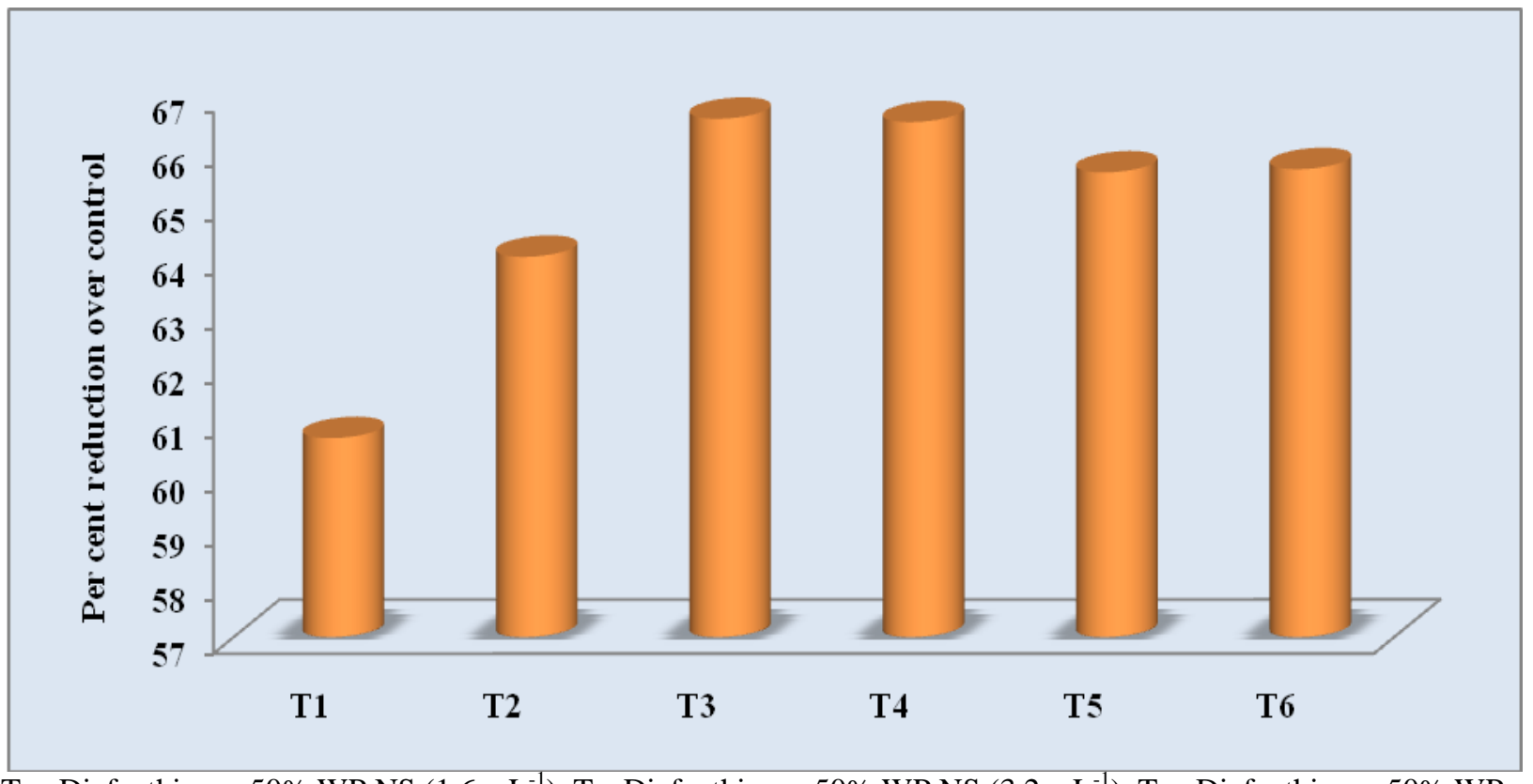

$\mathrm{T}_{1}$ - Diafenthiuron 50\% WP NS $\left(1.6 \mathrm{~g} \mathrm{~L}^{-1}\right), \mathrm{T}_{2}$ - Diafenthiuron 50\% WP NS $\left(3.2 \mathrm{~g} \mathrm{~L}^{-1}\right), \mathrm{T}_{3}$ - Diafenthiuron 50\% WP NS $\left(6.4 \mathrm{~g} \mathrm{~L}^{-1}\right), \mathrm{T}_{4}$ - Standard Diafenthiuron 50\% WP $\left(3.2 \mathrm{~g} \mathrm{~L}^{-1}\right), \mathrm{T}_{5}$ - Standard Quinalphos $25 \% \mathrm{EC}\left(7.2 \mathrm{ml} \mathrm{L}^{-1}\right), \mathrm{T}_{6}$ Standard Quinalphos $25 \%$ EC $\left(14.4 \mathrm{ml} \mathrm{L}^{-1}\right)$

Fig.3 Effect of diafenthiuron 50\% WP (NS) on capsule damage by $C$. punctiferalis(Location Nedukandam) - Third Application

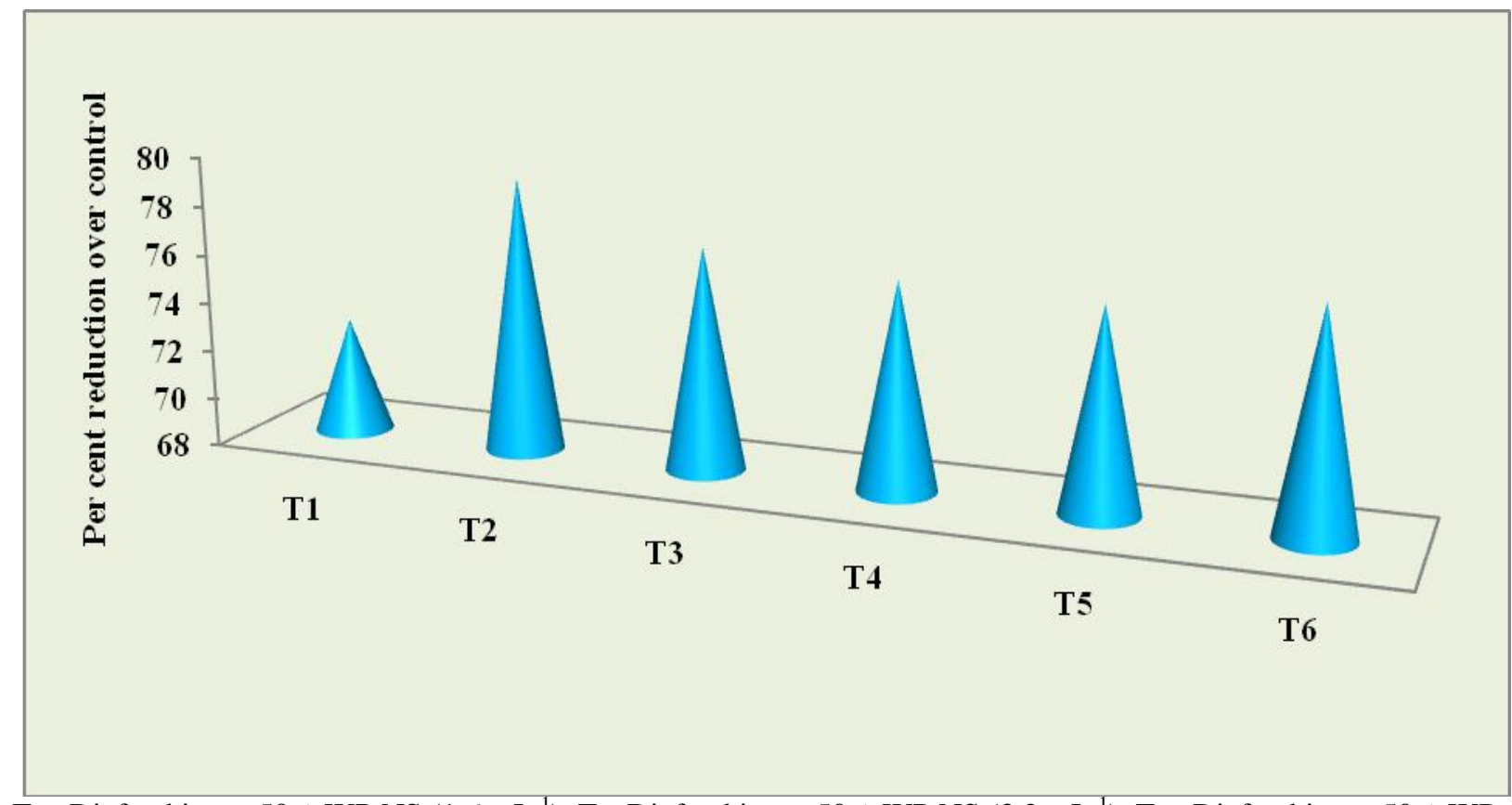

$\mathrm{T}_{1}$ - Diafenthiuron 50\% WP NS (1.6 $\left.\mathrm{g} \mathrm{L}^{-1}\right), \mathrm{T}_{2}$ - Diafenthiuron 50\% WP NS $\left(3.2 \mathrm{~g} \mathrm{~L}^{-1}\right), \mathrm{T}_{3}$ - Diafenthiuron 50\% WP NS (6.4g L $\left.\mathrm{g}^{-1}\right), \mathrm{T}_{4}$ - Standard Diafenthiuron 50\% WP $\left(3.2 \mathrm{~g} \mathrm{~L}^{-1}\right), \mathrm{T}_{5}$ - Standard Quinalphos $25 \% \mathrm{EC}\left(7.2 \mathrm{ml} \mathrm{L}^{-1}\right), \mathrm{T}_{6}$ Standard Quinalphos $25 \%$ EC $\left(14.4 \mathrm{ml} \mathrm{L}^{-1}\right)$ 
Fig.4 Effect of diafenthiuron 50\% WP (NS) on shoot damage by $C$. punctiferalis (Location -Nedukandam) - First Application

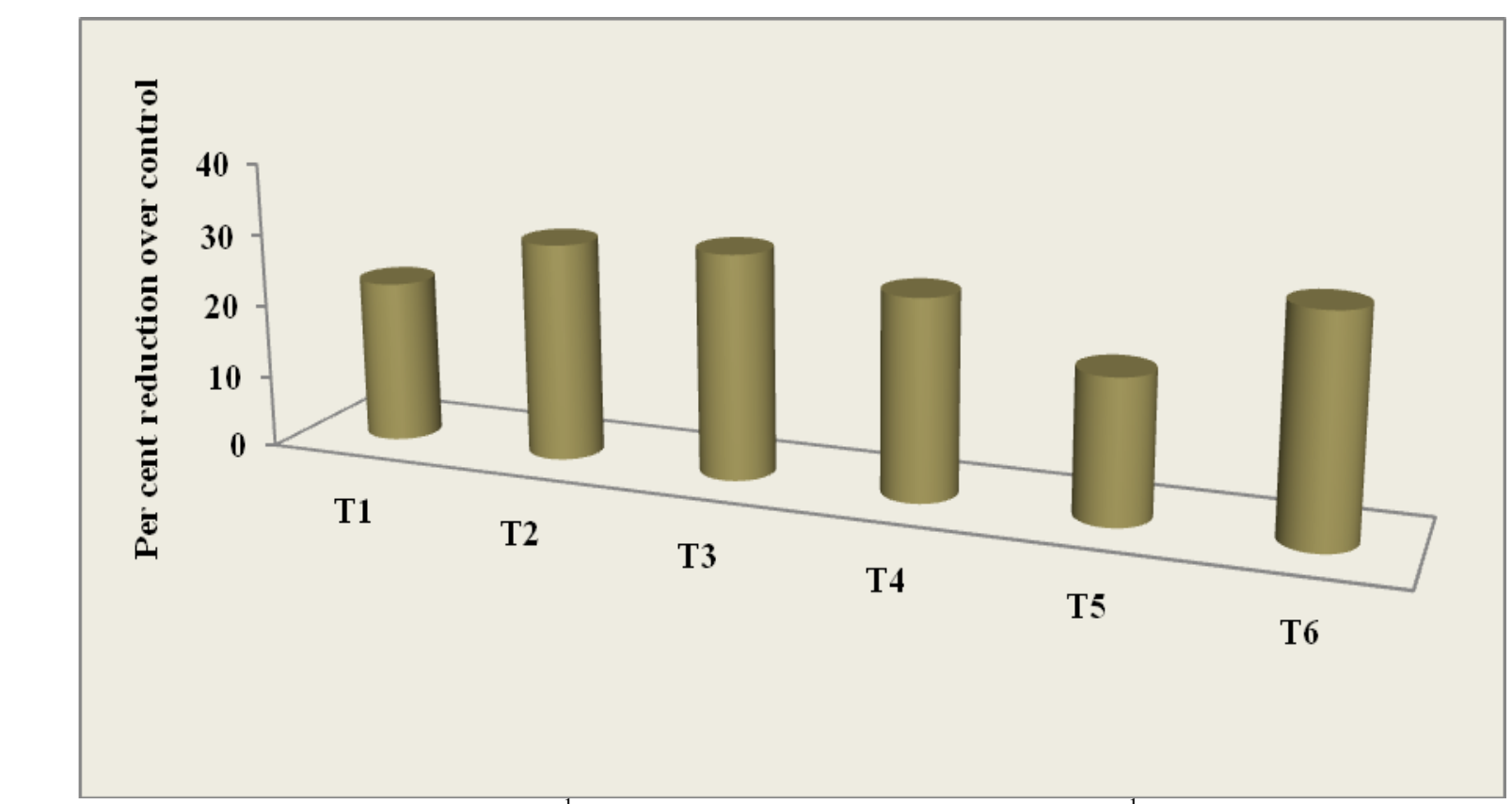

$\mathrm{T}_{1}$ - Diafenthiuron 50\% WP NS (1.6 g L $\left.\mathrm{g}^{-1}\right), \mathrm{T}_{2}$ - Diafenthiuron 50\% WP NS $\left(3.2 \mathrm{~g} \mathrm{~L}^{-1}\right), \mathrm{T}_{3}$ - Diafenthiuron 50\% WP NS (6.4g L $\left.\mathrm{g}^{-1}\right), \mathrm{T}_{4}$ - Standard Diafenthiuron 50\% WP $\left(3.2 \mathrm{~g} \mathrm{~L}^{-1}\right), \mathrm{T}_{5}$ - Standard Quinalphos $25 \% \mathrm{EC}\left(7.2 \mathrm{ml} \mathrm{L}^{-1}\right), \mathrm{T}_{6}$ Standard Quinalphos $25 \%$ EC $\left(14.4 \mathrm{ml} \mathrm{L}^{-1}\right)$

Fig.5 Effect of diafenthiuron 50\% WP (NS) on shoot damage by $C$. punctiferalis (Location -Nedukandam) - Second Application

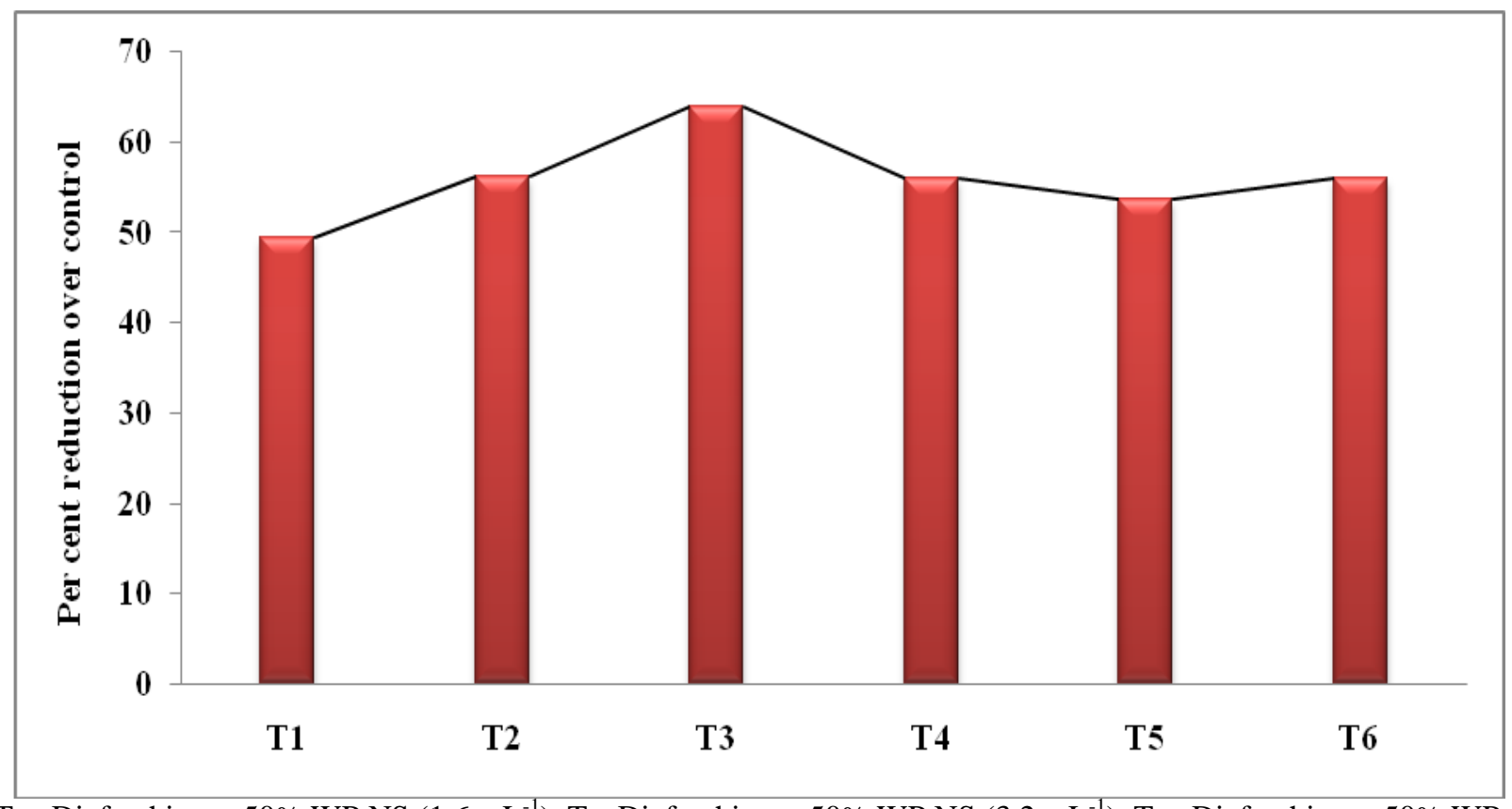

$\mathrm{T}_{1}$ - Diafenthiuron 50\% WP NS $\left(1.6 \mathrm{~g} \mathrm{~L}^{-1}\right), \mathrm{T}_{2}$ - Diafenthiuron 50\% WP NS $\left(3.2 \mathrm{~g} \mathrm{~L}^{-1}\right), \mathrm{T}_{3}$ - Diafenthiuron 50\% WP NS (6.4g L $\left.\mathrm{g}^{-1}\right), \mathrm{T}_{4}$ - Standard Diafenthiuron 50\% WP $\left(3.2 \mathrm{~g} \mathrm{~L}^{-1}\right), \mathrm{T}_{5}$ - Standard Quinalphos $25 \% \mathrm{EC}\left(7.2 \mathrm{ml} \mathrm{L}^{-1}\right), \mathrm{T}_{6}$ Standard Quinalphos 25\% EC $\left(14.4 \mathrm{ml} \mathrm{L}^{-1}\right)$ 
Fig.6 Effect of diafenthiuron 50\% WP (NS) on shoot damage by $C$. punctiferalis (Location -Nedukandam) - Third Application

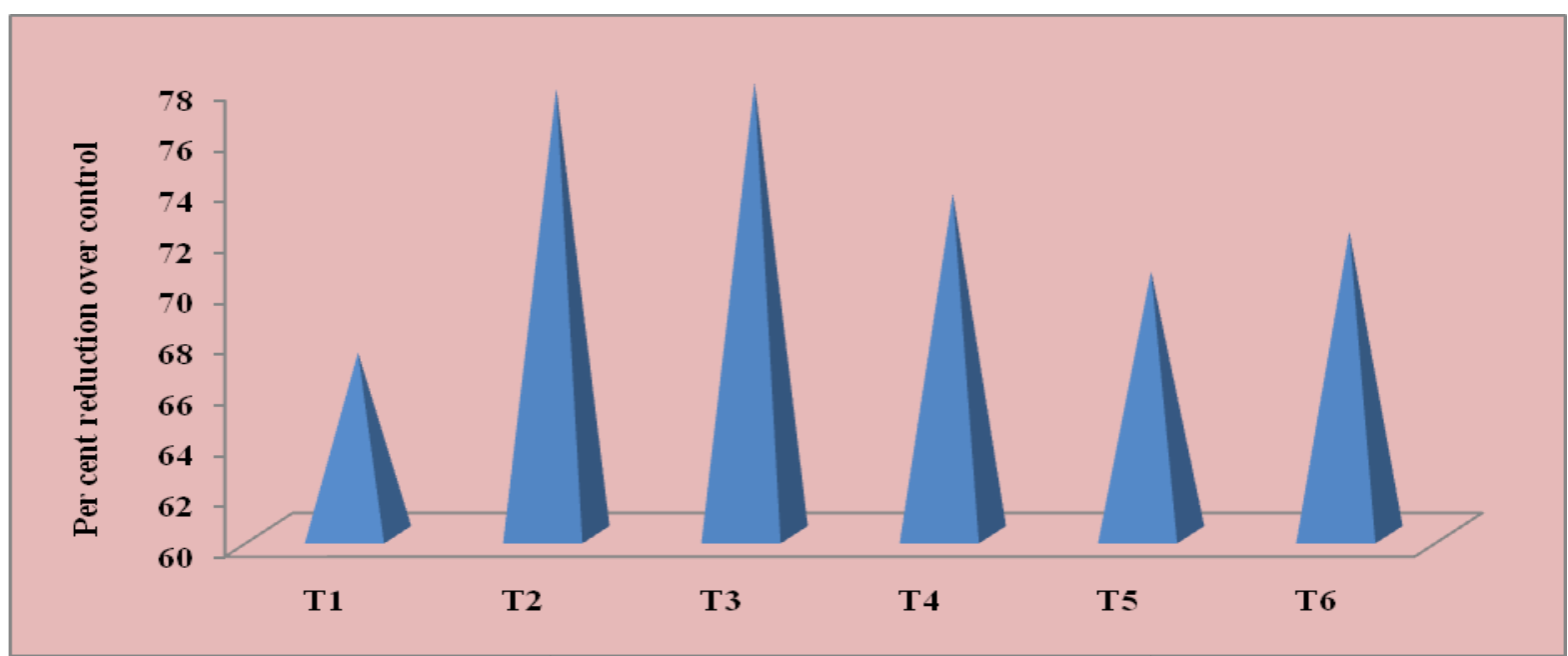

$\mathrm{T}_{1}$ - Diafenthiuron 50\% WP NS (1.6 $\left.\mathrm{g} \mathrm{L}^{-1}\right), \mathrm{T}_{2}$ - Diafenthiuron 50\% WP NS $\left(3.2 \mathrm{~g} \mathrm{~L}^{-1}\right), \mathrm{T}_{3}$ - Diafenthiuron 50\% WP NS $\left(6.4 \mathrm{~g} \mathrm{~L}^{-1}\right), \mathrm{T}_{4}$ - Standard Diafenthiuron 50\% WP $\left(3.2 \mathrm{~g} \mathrm{~L}^{-1}\right), \mathrm{T}_{5}$ - Standard Quinalphos $25 \% \mathrm{EC}\left(7.2 \mathrm{ml} \mathrm{L}^{-1}\right), \mathrm{T}_{6}$ Standard Quinalphos $25 \%$ EC $\left(14.4 \mathrm{ml} \mathrm{L}^{-1}\right)$

At 10 and 20 DAA after third spray, the damage ranged from 6.90 to 32.00 per cent and 6.90 to 8.50 per cent in diafenthiuron 50 WP (NS) treated plots. Diafenthiuron $50 \mathrm{WP}$ (NS) $800 \mathrm{~g}$ a.i. ha ${ }^{-1}$ per cent recorded the least damage of 20.00 per cent at 30 DAA which was on par with lower dose of $1600 \mathrm{~g}$ a.i. $\mathrm{ha}^{-1}$ (77.07\%) and diafenthiuron 50 WP (ES) 800 g a.i. ha ${ }^{-1}(76.41 \%)($ Fig 4,5 \&6). The order of efficacy of the chemicals in controlling the shoot damage in terms of per cent reduction over control was diafenthiuron $50 \mathrm{WP}$ (NS) $1600 \mathrm{~g}$ a.i. $\mathrm{ha}^{-1}$ per cent $(77.86 \%)>$ diafenthiuron $50 \mathrm{WP}$ (NS) $800 \mathrm{~g}$ a.i. ha ${ }^{-1}$ per cent $(77.63 \%)>>$ diafenthiuron $50 \mathrm{WP}(\mathrm{ES})$ $800 \mathrm{~g}$ a.i. $\mathrm{ha}^{-1}(73.47 \%)>$ quinalphos $25 \mathrm{EC}$ $1200 \mathrm{~g}$ a.i. $\mathrm{ha}^{-1}(71.98)>$ diafenthiuron $50 \mathrm{WP}$ (ES) $400 \mathrm{~g}$ a.i. $\mathrm{ha}^{-1}$ per cent $67.19 \%$ )(Table 6)

These findings were similar to that of Rajabaskar (2003), who found a cumulative reduction of 81.51 per cent in CSCB damage during 2002-2003 in Lower Palani Hills with diafenthiuron 0.16 per cent. Meanwhile, Stanley (2007) reported that diafenthiuron at 0.16 per cent recorded $61.85,86.72$ and 93.80 per cent reduction of capsule damage in trial conducted at Devarshola, Gudalur. Earlier reports on the effectiveness of diafenthiuron against lepidopteran pests like Plutella xylostella L.in cabbage (Ellis et al., 1992). Different formulations of quinalphos viz., AF, CS and EC tested by Valarmathi (1997) proved that quinalphos $25 \mathrm{EC}$ at 0.025 per cent effected only 14.3 per cent mean reduction on capsule damage by $\mathrm{CSCB}$, as against the highest of AF and CS.

Kubendran (2012) reported that, after three rounds of application, flubendiamide $240+$ thiacloprid $240-480 \mathrm{SC}^{\mathrm{RM}}$ at $7.2+7.2$ and $6.0+6.0 \mathrm{~g}$ a.i. $\mathrm{hl}^{-1}$, thiodiarb 70WP + thiacloprid $240 \mathrm{SC}^{\mathrm{TM}}$ at $42.0+6.0 \mathrm{~g}$ a.i. $\mathrm{hl}^{-}$ ${ }^{1}$ and flubendiamide $480 \mathrm{SC}$ at $6.0 \mathrm{~g}$ a.i. $\mathrm{hl}^{-1}$ recorded minimum shoot damage of CSCB ranging from 8.12 to $9.25,15.15$ to 16.79 and 11.11 to 14.59 per cent in I, II and III seasons respectively. Meanwhile, Stanley (2007) reported that diafenthiuron at 0.16 per cent recorded $61.85,86.72$ and 93.80 per cent reduction of capsule damage in trial conducted at Devarshola, Gudalur. Ranjith (2009) reported that the Diafenthiuron 50 WP (NS) at 800 and $400 \mathrm{~g}$ a.i. ha ${ }^{-1}$ proved its 
efficacy against CSCB and reduced the shoot damage level up to $82.39,85.46$ and 80.04, 84.95 per cent at both locations viz., Murukkadi and Onnamile respectively.

\section{References}

Chakravarthy, A. K., and M. M. Khan. 1987. Pests of cardamom. Cardamom 20: 1419

Ellis, W. W., Sribuddhacharat, A. R, Chaimongtal, I., Hare, C. J. and Teng, P. S. 1992. Diafenthiruon: Field studies with Plutella xylostella (L.) control of South East Asia. In: Proc. III ${ }^{\text {rd }}$ Intl. Conf. Plant Protec. in Tropics. Counting Highlands, Malaysia. pp. 6670.

Ishaaya, I., Mendelson, Z. and Horowitz, A. R. 1993. Toxicity and growthsuppression exerted by diafenthiuron in the sweetpotato whitefly, Bemisia tabaci. Phytoparasitica 21: 199-204.

Kubendran, D., 2011. Evaluation of flubendiamide + thiacloprid $\mathrm{SC}^{\mathrm{RM}}$ against Conogethes punctiferalis Guenee and Sciothrips cardamomi Ramk. On cardamom. Unpub Ph. D Thesis, Faculty of Agriculture and Animal Husbandry, Gandhigram Rural Institute, Gandhigram, Tamil Nadu. $162 \mathrm{p}$.

Kumaresan, D., Varadarasan, S. and Gopakumar, B. 1987. Review and current status of research on insect pest control in cardamom cropping system. J. Coffee Res., 17: 84-87
Murugan, M., P. K. Shetty, M. B. Iremath, R. Ravi and A. Subbiah. 2011. Occurence and activity of cardamom pests and honeybees as affected by pest management and climatic change. International Multidisciplinary Research Journal 1(6): 3-12.

Rajabaskar, D., 2003. Studies on the Evaluation of IPM Modules against Conogethes punctiferalis Guenee and Sciothrips cardamomi Ramk. On Cardamom. Unpub. Ph.D Thesis. Tamil Nadu Agric. Univ., Coimbatore, India. 198p.

Ranjith, M., 2012. Bioefficacy, Safety and Phytotoxicity of Diafenthiuron 50WP in Cardamom. Pub. Ph.D Thesis. Tamil Nadu Agric. Univ., Coimbatore, India. $95 \mathrm{p}$

Stanley, J., 2007. Chemical and Behavioural Approaches for Pest Management in Cardamom. Ph.D. Thesis. Tamil Nadu Agric.Univ, Coimbatore, India, 210 p.

Thyagaraj, N. E., 2002. Integrated Management of Some Important Cardamom Pests in Hill Region of Karnataka, South India. Unpub. Ph.D Thesis, Dr. B.R. Ambedkar University, Agra. 213p.

Valarmathi, S., 1997. Bioefficacy and Residues of Quinalphos Applied as Ecofriendly Formulations, Aquaflow (AF) and Colloidal Suspension (CS) on Cardamom and Cotton. Ph. D. Thesis, Tamil Nadu Agric. Univ., Coimbatore, India. 161p.

\section{How to cite this article:}

Aravind, J., K. Samiayyan and Kuttalam, S. 2017. A Novel Insecticide Diafenthiuron 50WP against Cardamom Shoot and Capsule Borer C. punctiferalis Guenee. Int.J.Curr.Microbiol.App.Sci. 6(10): 4995-5004. doi: https://doi.org/10.20546/ijcmas.2017.610.473 